\title{
Design and analysis of a new tactile device featuring Magneto-Rheological fluid in control force of robotic surgery
}

\author{
Le Tran Huy Thang, Do Xuan Phu*
}

MediRobotics Lab, Department of Mechatronics and Sensor Systems Technology, Vietnamese-German University, Vietnam

\section{Correspondence}

Do Xuan Phu, MediRobotics Lab, Department of Mechatronics and Sensor Systems Technology,

Vietnamese-German University, Vietnam

Email: phu.dx@vgu.edu.vn

\section{History}

- Received: 2018-12-26

- Accepted: 2019-04-22

- Published: 2019-06-30

DOI :

https://doi.org/10.32508/stdj.v22i2.1342

\section{Check for updates}

\section{Copyright}

(c) VNU-HCM Press. This is an openaccess article distributed under the terms of the Creative Commons Attribution 4.0 International license.

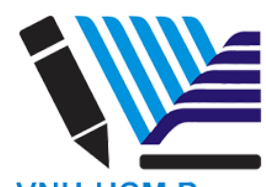

\begin{abstract}
In this paper, a new artificial skin tissue device which can emulate the stiffness of several organs of human is proposed and analyzed utilizing magneto-rheological (MR) fluid (MR skin). The proposed skin could be applied for the robot-assisted surgery manipulated by haptic devices as a controllable tactile sensor. The method in this paper is design of multi- embedded valve networks inside the structure of the master actuator. These valves use the flow mode and shear mode of MR for pressure control. Deformation equation of the MR skin is derived and the external force contacting the MR skin is also analyzed. After formulation, the proposed tactile display is optimized by using the finite element method software (ANSYS ADPL). It is shown via the optimization that the results can satisfy the initial requirements of the design. From the simulation results, the adjacent coils with similar setup show outstanding results compared with adjacent coils with discordant setup. This directly indicates that the proposed MR skin structure is feasible in the manufacturing and is applicable to haptic devices, especially those used for robotic surgery.

Key words: Magneto-rheological fluid, MR skin, MR actuator, Optimization, Pressure control, Tactile device, Deformation change
\end{abstract}

\section{INTRODUCTION}

Recently, many research studies have been carried out on mmicking human skin via electronics, as shown in Figure 1. Nonetheless, according to Wang et al., simulating the human skin using tactile display with the ability to distinguish among a wide range of force values and with a rapid response is still challenging ${ }^{1}$. Moreover, the device's ability to mimic the surface of an object, including the deformation, texture, shape, roughness and temperature, also warrant further research. By simulating the surface of the human organs, the device transmits small-scale information of the deformation of the skin to the operator's hand ${ }^{2}$. This application could be applied in minimally invasive surgery in order to provide a realistic environment for teletaction and high surgical precision. Such devices could be developed using different approaches, including electromagnetic technologies, shape memory alloys, electrorheological fluids, magnetorheological (MR) fluids and pneumatic systems ${ }^{3}$. Among these compliant tactile displays, MR fluid possesses outstanding properties since it has low cost, low power consumption, and a simple configuration - without any moving components ${ }^{4}$. MR fluid is a kind of smart fluid which contains ferromagnetic particles - mostly micrometer scale - and oil carrier ${ }^{5}$. In ambient conditions, MR fluid behaves like Newtonian fluid. When the free-flowing liquid is placed under a magnetic field, MR fluid immediately changes its density and transforms into a semi-solid state with a different mode of operations, including valve mode, squeeze mode and shear mode. These properties allow the user to control the behavior of the MR fluid by manipulating the magnetic field ${ }^{6}$. Therefore, MR fluid is applied in many different research fields and has contributed to creating cutting-edge technology devices, such as MR brake, MR damper and MR valve ${ }^{7,8}$.

In this paper, MR skin consists of a multi-valve system which deploys the behavior of MR fluid; the progress of optimal valve design is proposed. The objective of this study is to create a new tactile display device with the ability to mimic the surface of different human organs. In order to achieve that, the MR skin surface would have to mimic the deformation of the organ based on palpation force and surface deformation aspects. This device is designed to provide the user's hand with realistic sensation through appropriate palpation force and surface deformation. MR skin has promising potential to be applied in robotic masterslave systems, such as the da Vinci ${ }^{T M}$ Surgical System. Due to the development of the surgical robotic system, the need for a tactile display device for better tactile sensations and spatially distributed information is 
recognized. The structures of the next sections are organized as follows: Section 2 gives an overview of the MR skin configuration and the valve network design, Section 3 analyzes the desired parameters in order to emulate the real organs and also the displacement calculation, and Section 4 provides the optimization parameters and the results configuration.

\section{CONFIGURATION AND ANALYSIS}

\section{MR skin configuration \\ MR kin design}

To provide the best experience, the width and length of the device are chosen to fit the dimensions of the human hand. When touching the device, the vibration stimulus would be recorded and classified, based on different characteristics such as strength and location. This design has a total of 25 cells and can be divided into 2 types. The configuration is shown in Figure 2 , in which type 1 refers to cells containing only MR Fluid and type 2 refers to valve cells. These cells are arranged alternately, and each MR fluid cell is surrounded by 2 or 4 valve cells.

The MR fluid cell is able to exert forces normal to the MR skin surface against the applied forces. The strength of the palpation force can be controlled by the intensity of the magnetic flux. This feature is manipulated by the electromagnet which is placed under the MR skin surface. The coils setup can be adjusted to create clock-wise or counter clock-wise magnetic field lines.

At the initial state, the MR skin has a flat surface, as shown in Figure 3. The fluid pressure inside the device can be calculated as the sum of the initial pressure $p_{o}$ the laminar pressure, and the turbulent pressure of the MR flow. The initial pressure is chosen so that it would be close to the ambient pressure. The turbulent pressure only appears when the Reynold number of the MR fluid is larger than 2300. According to studies by Goncalves et al., if the MR fluid velocity is smaller than $40 \mathrm{~m} / \mathrm{s}$, then its maximum Reynold number is just over $1200^{9}$. This value is significant lower than the critical Reynold number. The fluid velocity in the tactile display is undoubtedly smaller than 40 $\mathrm{m} / \mathrm{s}$. Thus, there would be no turbulent pressure in the MR skin and the formula of the pressure inside the device can be written as (Equation (1)):

$$
p=p_{0}+p_{\text {laminar }}
$$

As shown in Figure 3, below the MR skin's surface is a suspension system. This system connects with the frame underneath to stabilize the structure. The cylinder frame could be used as an electromagnet to manipulate the palpation force. The blue parts inFigure 3 are made from magnetic material. There is a displacement sensor installed under each valve cell. The displacement sensor could also function as a force sensor. These sensors would provide accurate information about the position, strength of the applied force, and how long the force would last. The control system would be based on these signals to adjust the valve.

\section{Valve design}

As mentioned above, the MR skin consists of valve cells and MR fluid cell. The valve system includes 12 identical valve cells and $13 \mathrm{MR}$ fluid cells. The gap of the valve is small enough so that the MR fluid would work properly when the magnetic field is applied. This gap also removes the block phenomenon of the valve. The coil is placed inside the valve system in order to increase the magnetic field strength. The dimensions of the valve are shown in Table 1.

When the valve is open, the MR skin surface will deform with a low palpation force. The valve gap is 0.5 $\mathrm{mm}$, which is sufficient for the MR fluid to operate effectively and prevents the sudden deformation of MR skin. Theoretically, with a high enough current intensity, the valve would completely block the movement of the fluid among the MR cells. The valve's crosssection has an isosceles trapezoidal shape, as shown inFigure 4. The inner length of the isosceles is $24 \mathrm{~mm}$ and the outer length of the isoceles is $30 \mathrm{~mm}$, as shown inTable 1. Due to the simple structure, its execution requires fewer accessories and no moving parts. Besides, this configuration allows the valve to work more efficiently when the magnetic field is applied.

\section{Working principle}

The MR skin is a tactile display which mimics the human organ surface to support the operator of the haptic device. InFigure 5, when the operator's fingertip contacts with the MR skin, the MR skin will have a slight vibration and displacement. This vibration is recorded by a force sensor under the valve cell. This sensor also works as a displacement sensor and collects the information of the MR skin surface deflection. The signal is converted from analog to digital and fed into the controller. This controller would manipulate the current power. The current intensity will directly control the magnetic field. This magnetic field would act like an actuating signal for the valve network. The displacement sensors are placed under the MR valve measuring the real time deflection of the MR skin surface and feedback to the controller. This feedback loop would provide the controller with the information of the position, velocity and the palpation force generated by the MR skin. Hence, the MR 


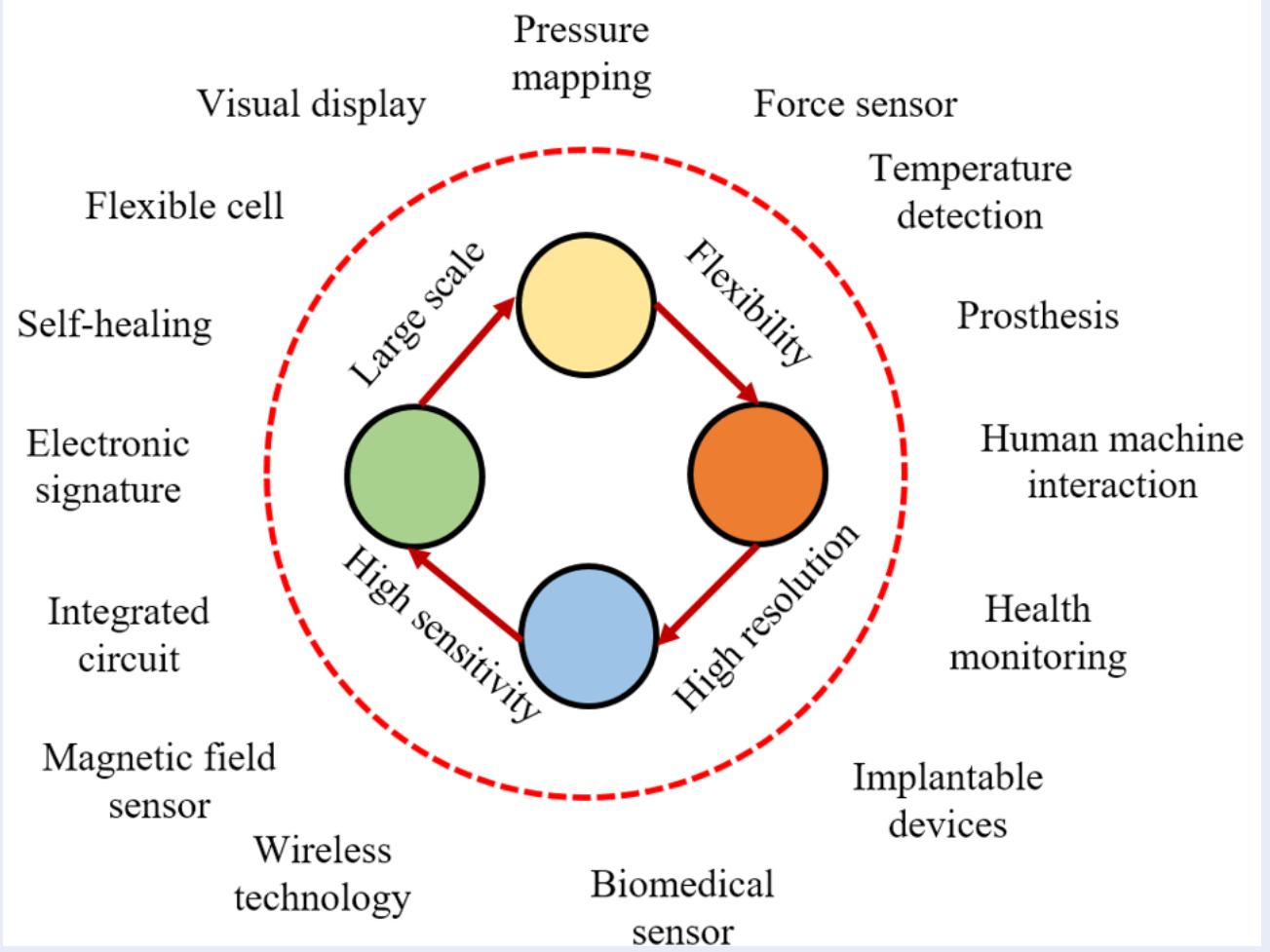

Figure 1: Recent applications of electronic skin technology.

\begin{tabular}{|c|c|c|}
\hline Parameter & Parameter unit & Value (mm) \\
\hline Gap thickness & & 0.5 \\
\hline Valve depth & & 30 \\
\hline Valve inner length & & 24 \\
\hline Valve length & & 30 \\
\hline Valve height & & 30 \\
\hline Coil height & & 6 \\
\hline Coil length & L3 & 24 \\
\hline
\end{tabular}

skin surface could maintain the deformation accurately and provide the user with a more realistic sensation.

\section{MR skin deformation analysis Desired palpation force}

To imitate the human organ, numerous factors need to be considered. Firstly, how the strength and frequency of the applied force affect the deformation are examined. Secondly, the environmental elements such as the temperature or texture of the organs are studied. At the initial stage of creating the MR skin, only the palpation force and the displacement of the MR skin will be analyzed. Palpation force is the reaction force between the MR skin and the human organ. A dynamic force sensor is used to measure this value. An experiment was carried out on five different external human organs ${ }^{2}$. The results are shown in Table 2 (by Han et al.) ${ }^{2}$. The palpation force oscillates approximately between 0.4 to $1.5 \mathrm{~N}$. Thus, MR skin needs to be able to exert a force normal to the tactile display surface with the value ranging from 0 to $2 \mathrm{~N}$. 


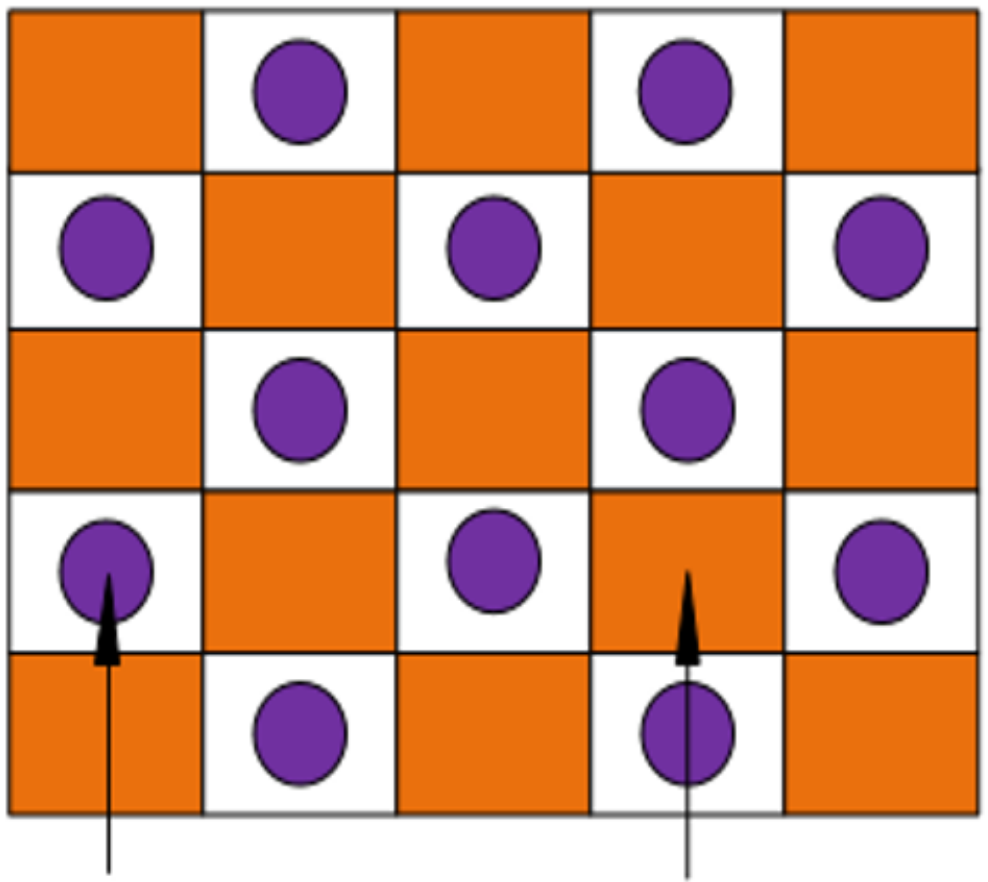

Valve

MR Fluid

Figure 2: MR skin top view.

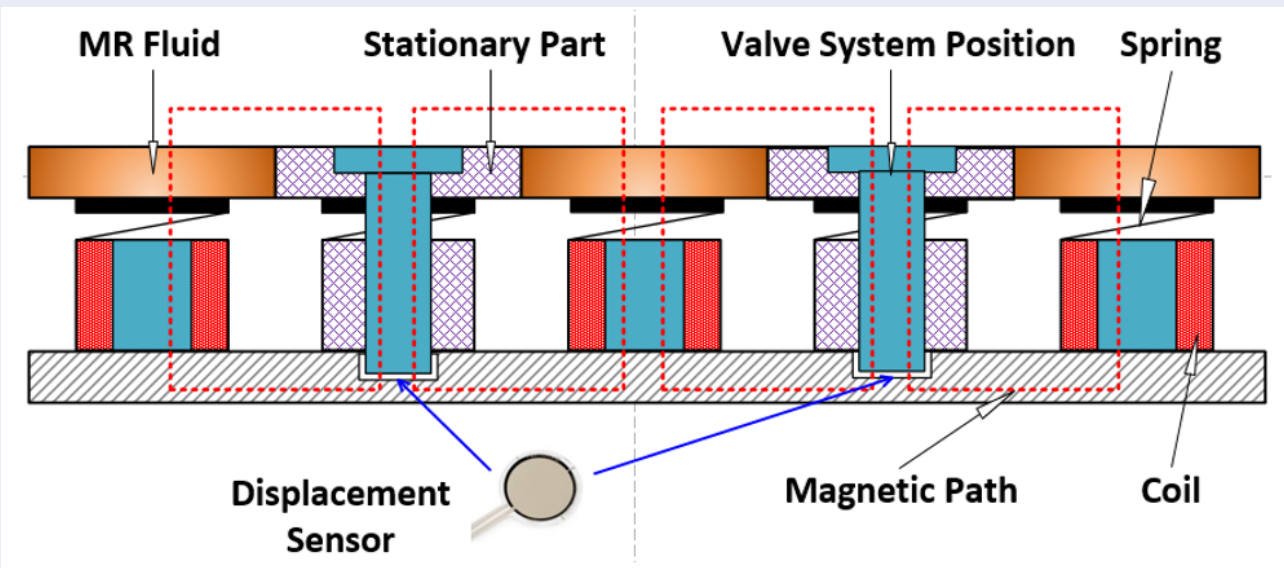

Figure 3: MRskin configuration 


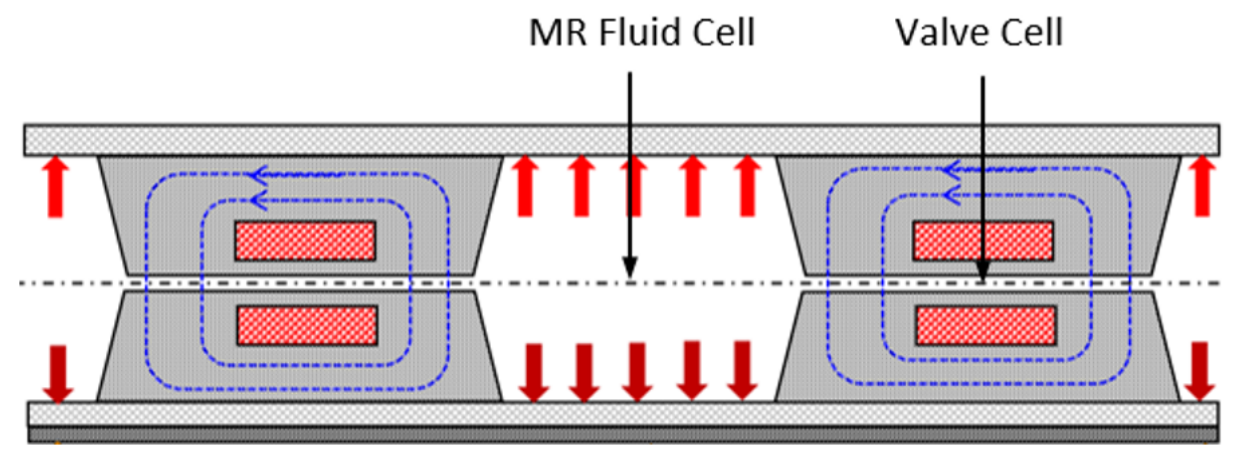

Figure 4: Design of the valve system in the MR skin.

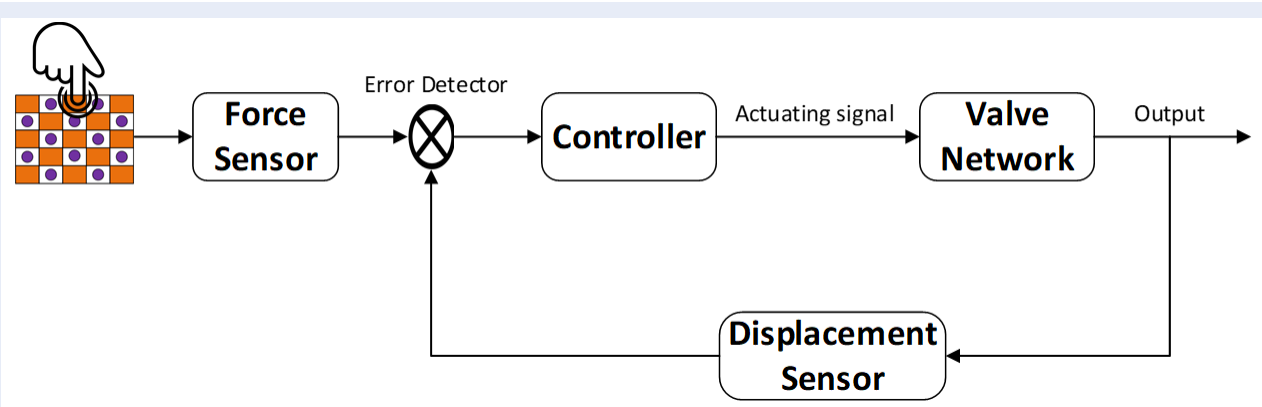

Figure 5: Control process diagram.

Table 2: Palpation force of 5 body parts.

\begin{tabular}{ll}
\hline Organ & Palpation Force $(\mathrm{N})$ \\
Hand & $0.6-0.9$ \\
Neck & $0.6-1.2$ \\
Abdomen & $0.4-1.3$ \\
Thigh & $0.7-1.2$ \\
Back & $1.0-1.5$ \\
\hline
\end{tabular}

\section{Desired displacement value}

To provide the realistic sensation when touching the MR skin, the displacement of the MR skin's surface also needs to be considered. In 2015, an experiment was carried out by Ogawa et al. on the stomach to evaluate the stiffness of the organ palpation device ${ }^{10}$. The results are shown in Figure 6. From these diagrams, a displacement value of approximately $0.004 \mathrm{~m}$ will generate a reaction force value around $1.2 \mathrm{~N}$. As mentioned above, the proposed device needs to produce a palpation value equal to $1.5 \mathrm{~N}$. Hence, to satisfy the design requirements, a displacement value of
$0.005 \mathrm{~m}$ was chosen.

\section{Desired elastic modulus}

Since the goal of the MR skin is to mimic the human organ, the elastic modulus of the MR skin also has to be considered. The material of the MR skin surface has to possess similar biomechanical properties as the human skin. The young modulus of the human skin could be found by using elastography. This is a medical imaging modal which shows the relationship between the elastic properties and the stiffness of soft tissue. 


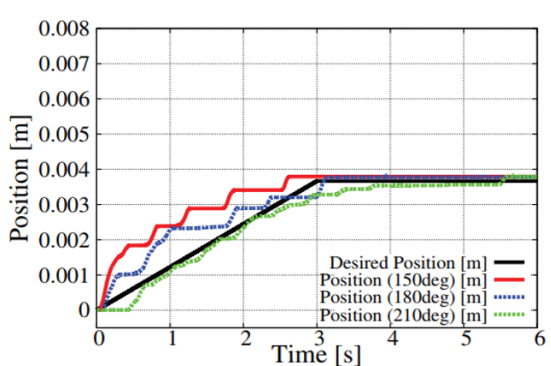

(a)

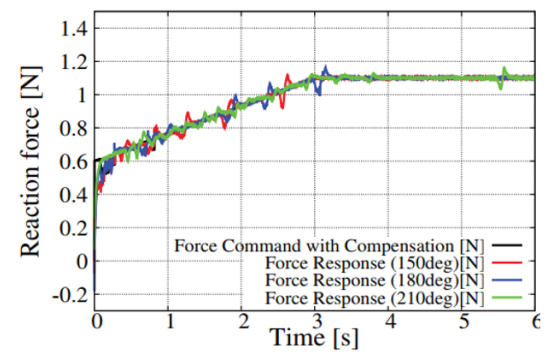

(b)

Figure 6: Organ displacement when applying force: (a) Position response, (b) Force response ${ }^{10}$.

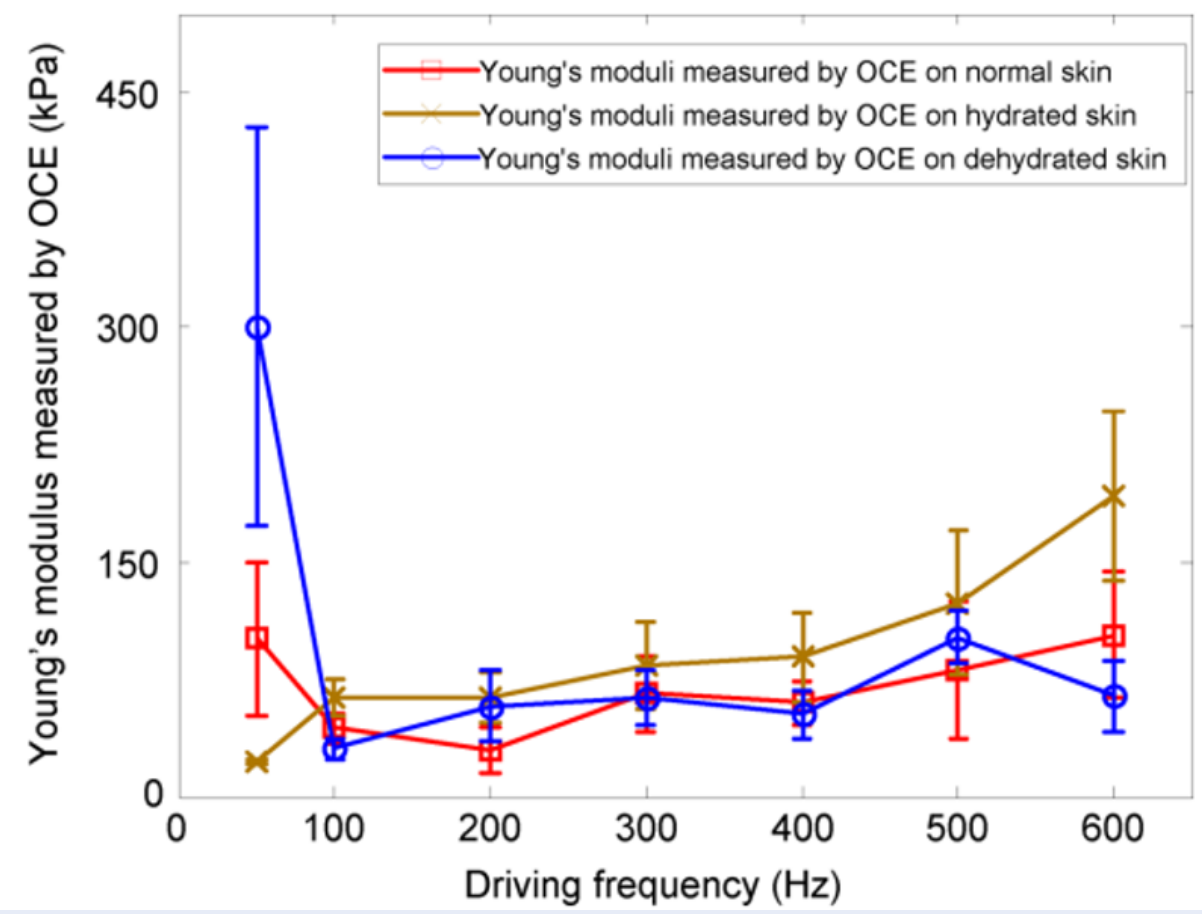

Figure 7: Young's modulus measured by OCE method under different driving frequencies and skin hydration conditions. Blue line denotes results from dehydrated skin, brown line denotes those from hydrated skin, and red line from normal skin ${ }^{11}$.

Xing Liang et al. had performed an experiment using dynamic optical coherence elastography (OCE) and mechanical surface wave propagation principle to measure the biomechanical properties of the human skin $^{11}$. The results are shown in Figure 7. The maximum Young modulus value of the human skin in this experiment was nearly $450 \mathrm{kPa}$. However, with the limitation of the material choice, it is very difficult to manufacture the MR skin surface which has such a low Young modulus like the human skin. Therefore, a material which has the Young modulus around $1 \mathrm{MPa}$ could be more suitable.

\section{MR skin surface deformation formula}

At the initial state, a high current intensity is used to activate the magnetic field. The relation between the magnetic field $\mathrm{B}$ and the current intensity $I_{c}$ is (Equation (2)):

$$
B=\mu_{0} n I_{c}
$$

Where $\mu_{0}$ is vacuum permeability which is equal to $4 \pi \cdot 10^{-7}(\mathrm{H} / \mathrm{m})$ and $\mathrm{n}$ is the number of coil turn. 


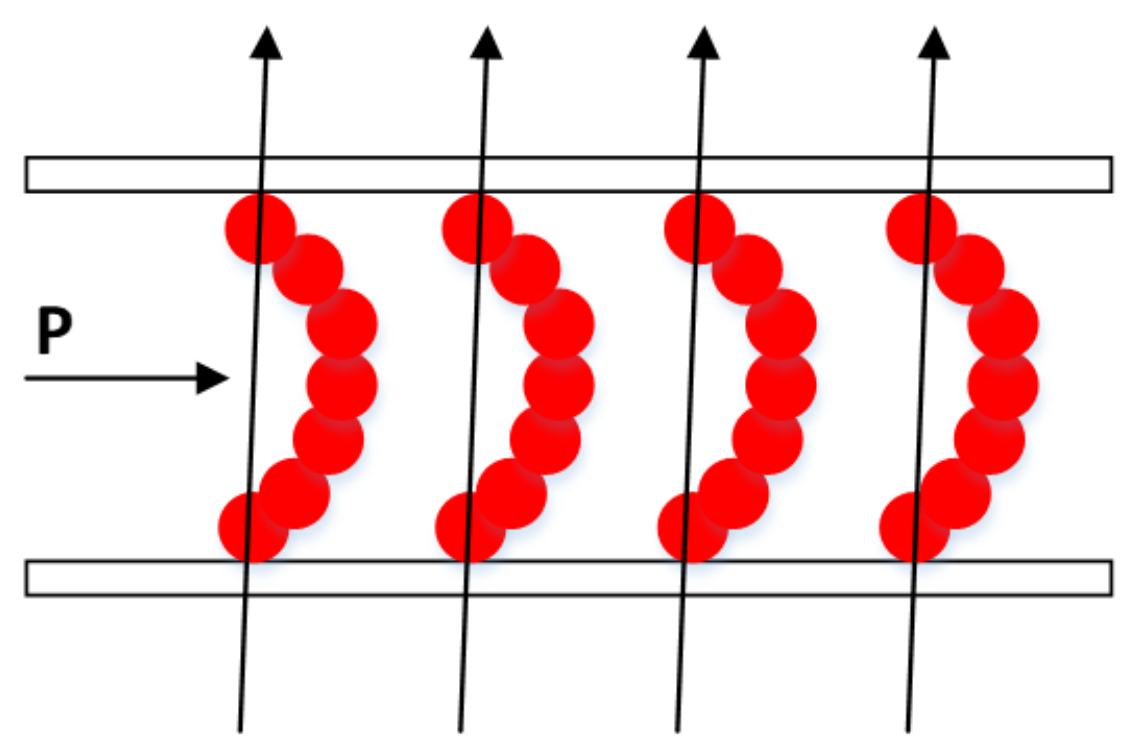

Figure 8: Flow mode of MR fluid.

The fluid inside and near the region of the valve cells would be in flow mode, as shown in Figure 8. Chain structures are formed along the magnetic force line under the effect of the magnetic field. Thus, the MR fluid could not flow through the valve until the pressure difference between the outer and inner surface is larger than the pressure created by the yield stress of the MR fluid. At the same time, the liquid in the MR fluid cell not affected by the magnetic field would behave like Newtonian fluid. In this case, gradually decreasing the magnetic field strength would result in change in the yield stress of the MR fluid. Hence, this would change the speed of the displacement and the reaction force of the MR skin when the user touches the surface. The volume flow $\mathrm{Q}$ of the liquid through the valve during the transition of MR fluid from liquid to solid is calculated as ${ }^{12}$ (Equation (3)):

$$
\begin{aligned}
& Q=\frac{\pi d}{24 m}\left(\frac{l}{\Delta p}\right)^{2} \\
& {\left[20 \tau_{y}^{3}-\frac{12 \Delta p}{l} \tau_{y}^{2}+15 h^{2}\left(\frac{\Delta p}{l}\right)^{2} \tau_{y}+2 h^{3}\left(\frac{\Delta p}{l}\right)^{3}\right]}
\end{aligned}
$$

Where $\tau_{y}$ is the critical shear yield stress of MR fluid and $\mathrm{m}$ is the apparent viscosity. For the simplicity of calculation, it is assumed that the force applied by the user's hand is spread equally over the MR fluid cell. Besides, only the deformation of one cell's surface would be analyzed. The differential equation of the forth order of the deflection curve could be calculated as follows ${ }^{13}$ (Equation (4)):

$$
E I D^{I V}=q
$$

Where $D$ is the displacement, $I$ is the moment of inertia, E is elastic modulus of the MR skin's surface, and $\mathrm{q}$ is the distributed load. From integration of Equation (4) four times, we have the equation below (Equation (5)):

$$
E I D=\frac{1}{24} q x^{4}+\frac{1}{6} C_{1} x^{3}+\frac{1}{2} C_{2} x^{2}+C_{3} x+C_{4}
$$

This equation is independent of support. If one plugs in the boundary conditions to solve for $C_{1}, C_{2}, C_{3}$ and $C_{4}$, the displacement of the skin's surface can be derived as follows (Equation (6)):

$$
D=\frac{p A l^{3}}{24 E I}\left[\left(\frac{x}{l}\right)^{4}-2\left(\frac{x}{l}\right)^{3}+\frac{x}{l}\right]
$$

To pajmic the human tissue, the elastic modulus could be calculated using Equation (7) ${ }^{14}$. This value is chosen so that it is close to the chosen value in section 3.2.

$$
E=\frac{2\left(1-v^{2}\right) \alpha q}{w}
$$

Where $v$ is the Poisson's ratio, $\alpha$ is the thermal expansion coefficient, $q$ is the intensity of the continuously distributed load, and $w$ is the component of displacement. 


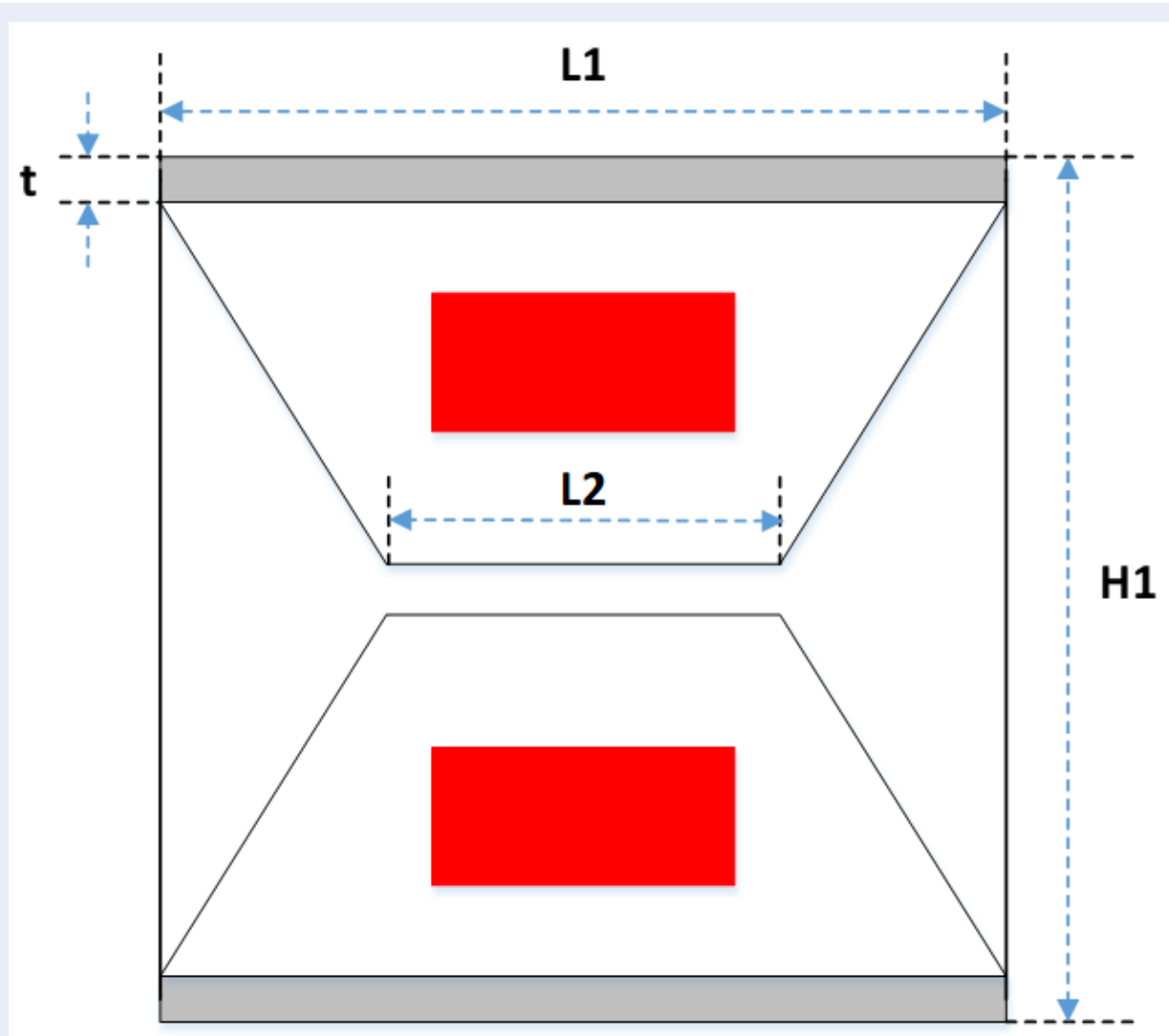

Figure 9: Configuration of the valve cell.

\section{OPTIMIZATION AND RESULTS}

In this design, a valve network is embedded inside the tactile display to provide the best experience for the user. The deformation of the MR skin and the elastic modulus of the MR skin surface material can imitate the characteristics of the human organ. The initial parameter and configuration are shown in Table 3 and Figure 9, respectively.

The software ANSYS 12 is used to solve the magnetic loop and program for the optimization. The process of optimizing the MR skin design concentrates around four parameters related to the dimension of the MR skin surface and MR fluid cell. These parameters have a significant role in determining the flow of MR fluid through the valve cell and the displacement of the MR skin when the user touches its surface. Table 4 shows the range and the final results of the optimization. With these parameters, the displacement and the palpation force value equivalent to $5 \mathrm{~mm}$ and $2 \mathrm{~N}$, respectively, could be achieved. The velocity of the deformation could be controlled by the current power. Besides, the coil's arrangement also affects the MR fluid behavior. Figure 10 shows the magnetic field inside the network with discordant coil setup. From the simulation, the first configuration clearly shows better results.

Different values of intensity currents are also carried out for the test. In Figure 10, a current value of 2 Ampere is used. Figure 10a shows a strong magnetic field (the green part) in the gap. Since the coil configuration in Figure 10a is better than Figure 10b, the first configuration is used to analyze the difference of the magnetic field when adjusting the current. In this case, two current intensity values equivalent to 1 and 0.5 Ampere, respectively, are used and shown in Figure 11. From Figure 10 and Figure 11, it is undeniable that the lower current would lead to a weaker magnetic field. With this relation, the gap could act like a throttle valve of the combustion engine. Thus, the amount of the fluid through the gap and its velocity could be controlled effectively.

From the above results, the objective of the design of MR skin was obtained. MR skin was analyzed for both configuration and applied force. The flow of MR 
Table 3: Initial optimized parameter.

\begin{tabular}{|c|c|c|}
\hline Parameter & Parameter unit & Initial Value $(\mathrm{mm})$ \\
\hline Surface thickness & & 0.5 \\
\hline Cell depth & & 30 \\
\hline Valve length & & 30 \\
\hline Valve height & & 30 \\
\hline
\end{tabular}

Table 4: Optimized parameters

\begin{tabular}{llll}
\hline Parameter & Lower Boundary $(\mathbf{m m})$ & Upper Boundary $(\mathbf{m m})$ & Optimized Value $(\mathbf{m m})$ \\
& 0.1 & 1.5 & 0.121 \\
& 20 & 50 & 40 \\
L1 & 24 & 50 & 24.01 \\
& 20 & 30 & 25 \\
\hline
\end{tabular}

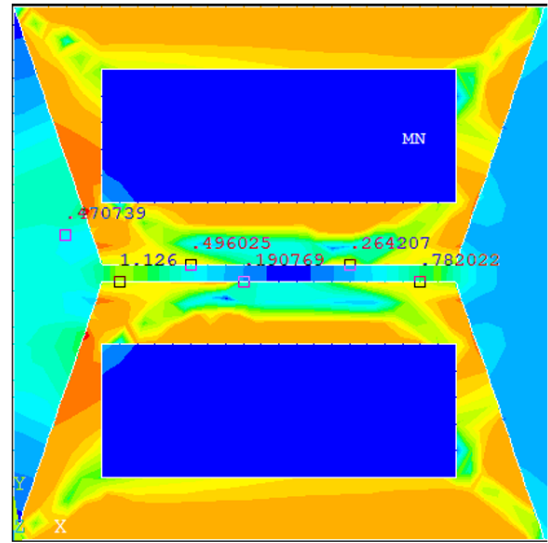

(a)

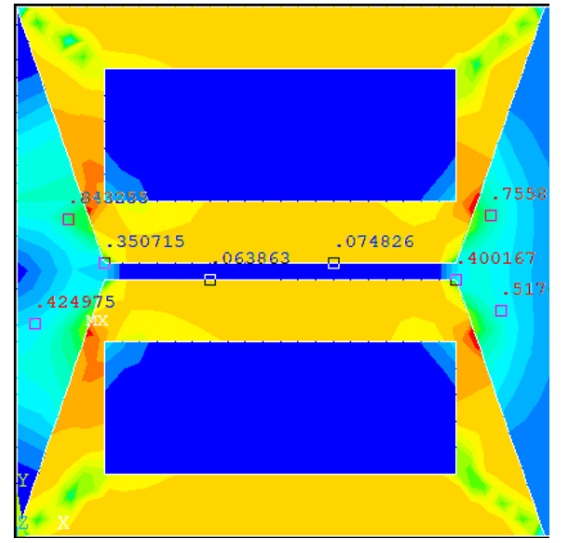

(b)

Figure 10: Magnetic flux density of the valve: (a) adjacent coils with similar setup (b) adjacent coils with discordant setup.

fluid inside the MR valve was optimized to prevent the block phenomenon. Based on the applied force on the surface of MR skin, the applied current can be adjusted to bring a comfortable sense for the human hand using control tactile devices. In addition, the simple structure and high efficiency in operation are also advantages of the proposed design, and could be optimized further for cost of manufacturing in the future.

\section{CONCLUSION}

In this study, the proposed device successfully reproduced the deformation of the human organ, especially with respect to displacement and palpation force aspects. A deformation of $5 \mathrm{~mm}$ and a palpation force of $2 \mathrm{~N}$ was achieved by the MR skin. Through simulation and optimization, surface thickness and cell depth should be equivalent to $0.121 \mathrm{~mm}$ and $40 \mathrm{~mm}$, respectively. Moreover, valve length and valve height equivalent to $24.01 \mathrm{~mm}$ and $25 \mathrm{~mm}$, respectively, are required to achieve the desired displacement and the desired palpation force. The valve network and the MR fluid inside could be used to control the speed and the depth of the surface deformation through magnetic field manipulation. It has been demonstrated that the pressure of MR valve can be controlled efficiently and, hence, MR skin is feasible to be applied in biomedical devices, such as haptic devices. For example, MR skin could be employed to incorporate with minimally invasive surgical technology to en- 


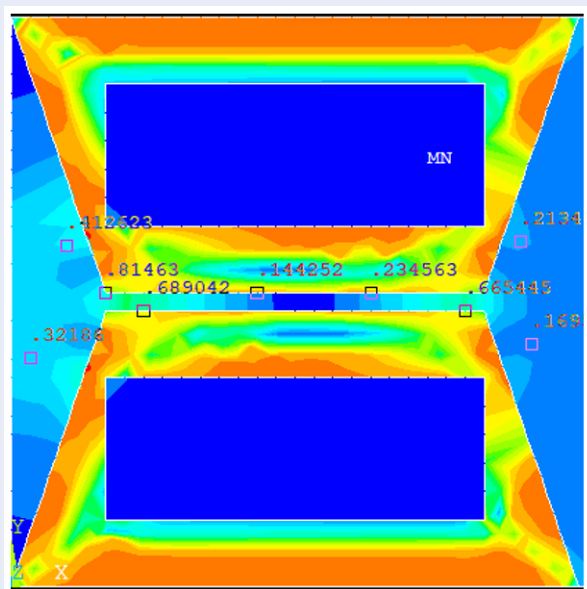

(a)

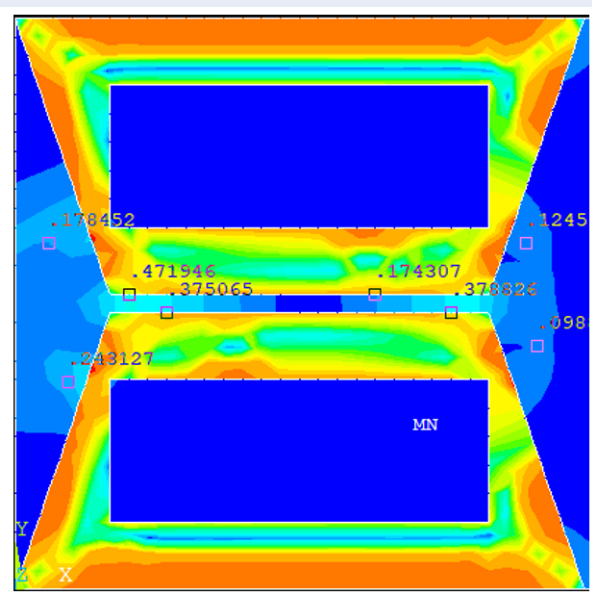

(b)

Figure 11: Magnetic flux density of the valve when: (a) I = 1 Ampere, (b) I = 0.5 Ampere.

hance the sensation of the operator. Moreover, mechanisms to control the velocity of the fluid flow through the valve under the magnetic field should be further studied. As well, investigations of the temperature and texture aspects of the MR skin need to be further explored for use in practical environment.

\section{ACKNOWLEDGMENTS}

This research is funded by the Ministry of Education and Training (Vietnam) under grant number B2018VGU-07 (KYTH-36). This financial support is gratefully acknowledged.

\section{COMPETING INTERESTS}

The authors declare that there is no conflict of interest.

\section{AUTHORS' CONTRIBUTIONS}

Do Xuan Phu conceived the design; Le Tran Huy Thang developed optimized the idea of design; Do Xuan Phu and Le Tran Huy Thang analyzed the data and wrote the paper.

\section{NOMENCLATURE}

$\mathrm{p}_{o}$ : Initial pressure of valve

plaminar: Laminar pressure of valve

g: Thickness of gap

d: Depth of valve

$\mathrm{L}_{2}$ : Inner length of valve

$\mathrm{L}_{1 \text { : }}$ Length of valve

$\mathrm{H}$ : Height of valve

$\mathrm{H}_{1}$ : Height of coil

$\mathrm{L}_{3}$ : Length of coil

B: Magnetic field
$\mathrm{I}_{c}$ : Current intensity

$\mu_{0}$ : Vacuum permeability

n: Number of coil turns

Q: Volume flow

$\tau_{y}$ : Critical shear yield stress of MR fluid

$\mathrm{m}$ : Apparent viscosity

D: Displacement

I: Moment of inertia

E: Elastic modulus of the MR skin's surface

q: Distributed load (intensity of the continuously distributed load)

v: Poisson's ratio

$\alpha$ : Thermal expansion coefficient

w: Component of displacement

\section{REFERENCES}

1. Wang X, Dong L, Zhang H, Yu R, Pan C, Wang ZL. Recent Progress in Electronic Skin. Adv Sci (Weinh). 2015;2(10):1500169. PMID: 27980911. Available from: 10.1002/advs.201500169.

2. Han YM, Oh JS, Kim JK, Choi SB. Design and experimental evaluation of a tactile display featuring magnetorheological fluids. Smart Mater Struct. 2014;23(7):077001. Available from: 10.1088/0964-1726/23/7/077001.

3. Chouvardas VG, Miliou AN, Hatalis MK. Tactile displays: overview and recent advances Author. Displays. 2008;29(3):185-94. Available from: 10.1016/j.displa.2007.07. 003.

4. Liu Y, Sun H, Leng J. Structural optimization of tactile display activated by magnetorheological fluid. Proc ICCM. 2007;16.

5. Bajkowski J, Skalski P. Analysis of viscoplastic properties of a magnetorheological fluid in a damper. Acta mechanica et automatica. 2012;6(3). Available from: http://www.actawm.pb.edu.pl/volume/vol6no3/BAJKOWSKI_ SKALSKI_EN_2011_021.pdf.

6. Choi SB, Li W, Yu M, Du H, Fu J, Do PX. State of the art of control schemes for smart systems featuring magneto-rheological materials. Smart Mater Struct. 2016;25(4):043001. Available from: $10.1088 / 0964-1726 / 25 / 4 / 043001$. 
7. Phu DX, Long MBQ, Chi LV, Thang LTH. Dynamic Analysis of Knee-Exoskeleton and Its implementation to Design of Magnetorheological Damper. Science and Technology Development Journal. 2018;21(1):002-14. Available from: 10.32508/ stdj.v21i1.421.

8. Do XP, Duc HT, Tran HTL, Choi SB, Yoon D. Design of a new inside multi-coils clutch for knee-exoskeleton structure based on Helmholtz phenomenon and magneto-rheological fluid. Proc SPIE. 2018;10598:105983. Available from: 10.1117/12. 2295662.

9. Goncalves FD. Characterizing the behavior of magnetorheological fluids at high velocities and high shear rates. PhD Thesis, Virginia Polytechnic Institute and State University, (2005):

10. Ogawa K, Ohnishi K. Evaluation of stiffness calculation for organ palpation device with electro-hydraulic transmission system based remote actuator. In: Proc. INDIN . vol. 13; 2015. p.
378-383. Available from: 10.1109/INDIN.2015.7281764.

11. Liang X, Boppart SA. Biomechanical properties of in vivo human skin from dynamic optical coherence elastography. IEEE Trans Biomed Eng. 2010;57(4):953-9. PMID: 19822464. Available from: 10.1109/TBME.2009.2033464.

12. Ishizuka $\mathrm{H}$, Lorenzoni N, Miki N. Characterization of Tactile Display for Stiffness Distribution Using Magneto-rheological Fluid. In: Proc. ICEP; 2014. p. 400-404. Available from: 10 1109/ICEP.2014.6826717.

13. Gross D. Dietmar Gross, Werner Hauger, Jörg Schröder, Wolfgang A. Wall, Javier Bonet, "Engineering Mechanics 2: Mechanics of Materials", Springer, (2011);:

14. Wu R, Lin W, Tang W, Liang F, Lin J. The Simulation Analysis on Performance of Magnetorheological Fluid Throttle Valve. Proc IEEE. 2011;p. 6529-32. Available from: 10.1109/RSETE. 2011.5965854 